Learning Whether to Cooperate in Novel Social Interactions

William H.B. McAuliffe ${ }^{1}$, Maxwell N. Burton-Chellew ${ }^{2}$, and Michael E. McCullough ${ }^{1}$

${ }^{1}$ Department of Psychology, University of Miami

${ }^{2}$ Department of Ecology and Evolution, University of Lausanne 


\begin{abstract}
Human social life is rife with uncertainty. In any given social interaction, one can wonder: Is cooperating with this person in my long-term best interest? Many people resolve to play it safe by cooperating rather than behaving selfishly, likely because (a) most social interactions in everyday life have long-term consequences and (b) the costs of alienating oneself from long-term social partners often outweighs the short-term benefits of cheating them. However, since trusting and sharing with others does not always advance self-interest, people might also learn through experience whether cooperation benefits them in any particular situation. Here, we review several lines of evidence suggesting that people initially decide when to share and trust based on the incentives that are present in their daily lives, but that they can also learn through experience to adjust their cooperation decisions to match the incentives of novel situations.
\end{abstract}

Keywords: cooperation, prediction error, economic games, trust, habit 
People leave tips at restaurants to which they never to plan to return, post reviews of hotels they will never visit again, and share money with strangers in anonymous laboratory experiments. What explains humans' propensity to help others, even when they do not know them and are unlikely to ever meet them again? One promising explanation is that our modernday decency reflects the importance of maintaining social partners in our evolutionary past (Krasnow \& Delton, 2016; Raihani \& Bshary, 2015). This theory suggests that a hard-wired tendency to cooperate in novel social interactions is operative today because in the past the risk of alienating a potential long-term social partner typically outweighed the short-term benefits of behaving selfishly.

However, it is not always in a person's long-term self-interest to cooperate: Some social partners prove untrustworthy, the material benefits of selfishness occasionally do outweigh the costs of angering a social partner, and sometimes it really is unlikely that two people will ever have the chance to interact again. Thus, natural selection may have also favored cognitive mechanisms that adjust decisions based on whether cooperation promotes self-interest within specific situations. Specifically, an ability to learn through personal experience or observing others whether behaving generously in a certain type of situation is rewarded - and whether behaving selfishly is punished — would allow people to make informed decisions about whether to cooperate in analogous future situations. An emerging literature suggests that people can override default tendencies to cooperate if they have opportunities to habituate to situations in which cooperation does not advance self-interest (Rand et al., 2014).

Researchers have relied on the concept of a prediction error to explore the role of learning in cooperation (FeldmanHall \& Dunsmoor, 2018). A prediction error represents the discrepancy between the expected outcome of a decision (e.g., I believe that cooperating in this 
situation will be good for my reputation in the long-term) and the actual outcome (e.g., it turns out that cooperating had no long-term effect on my reputation). By incorporating past prediction errors in their decision-making, people can choose whether to cooperate based on the presence or absence of situational factors that have been correlated with desired outcomes in the past.

\section{Learning to (Not) Share}

One type of situation in which prediction errors are likely to influence future behavior are those in which it is never in one's self-interest to cooperate. For example, many economic games require laboratory participants to decide whether to share windfalls of money with anonymous strangers in one-shot interactions (i.e., in which they will never knowingly interact with the anonymous strangers again). Economic games present situations that differ greatly from those of everyday life, where people typically decide whether to share resources with others they already know or might interact with again. Thus, although cooperation in economic games could reflect a desire to benefit anonymous strangers, it could also reflect the fact that participants have yet to register the mismatch between the situations they are used to and the economic game situation.

To illustrate this mismatch, consider the debate over the existence of so-called conditional cooperators, who choose whether to cooperate based on whether they believe others will cooperate too. Researchers have inferred the existence of conditional cooperators from experiments in which people play a public goods game, where members of a group can contribute money to a common resource that benefits everyone equally. In the game, each dollar contributed benefits the group because the experimenter multiplies all contributions by a constant $(M>1)$ before sharing them out equally. Thus, each dollar contributed returns $M$ dollars to the group, and $M / N$ dollars to each member. However, because $M$ is typically set to less than the number of group members $(N>M>1)$, each dollar contributed is personally costly $(N / M<1)$. 
Therefore, contributing is cooperative because it benefits others at a personal cost. The canonical result is that conditional cooperators contribute much of their endowment in early rounds, but by the end contribute little if anything (for a review, see Chaudhuri, 2011).

Researchers typically posit that conditional cooperators want to promote the public good, but eventually stop contributing out of disgust with free-riders who consistently contribute nothing. In essence, the conditional cooperation hypothesis posits that individuals perfectly understand the game, but must learn whether their counterparts share their goals. The conditional cooperation hypothesis is challenged, however, by the fact that many conditional cooperators later report that they were trying to maximize their income during the experiment, a motivation that is at odds with the consequences of their decisions (Burton-Chellew, El Mouden, \& West, 2016). Furthermore, the same decline in cooperation occurs when participants play with computers rather than humans (Burton-Chellew \& West, 2013; Houser \& Kurzban, 2002). Thus, declining contributions in these cases cannot reflect a prosocial regard for others that is eroded by exasperation toward non-cooperators. Instead they likely reflect a mistaken belief that contributions can increase personal income depending upon whether other participants also contribute, a confusion that is corrected by experience.

Further support for the confusion hypothesis comes from a study where participants played a public goods game with humans but could observe the contributions of people in other groups only, not in their own (Burton-Chellew, El Mouden, \& West, 2017). In these cases, participants mimicked the behaviors of successful players in other groups by discontinuing their contributions in their own groups. Subsequently, participants played a new public goods game with computers instead of humans. Those that had observed successful players in games with humans contributed less toward the computers than did those that had not observed successful 
human players, indicating that the former group had learned how to maximize their income better than had the latter group.

The learning perspective on human cooperation suggests that participants in economic games start out believing that cooperation promotes self-interest because they are importing the behaviors that have been rewarded in their everyday lives to novel situations. For example, participants from societies where interactions among strangers are effectively regulated by law cooperate more in economic games (Stagnaro, Arechar, \& Rand, 2017). If people really do impose these mental models upon the experimental games they encounter in the lab, the meaning of subjects' behavior in experimental games becomes problematic because we cannot know with certainty which outside frame of reference participants are using to interpret the game. Instead, the same economic game may evoke different working models from real-life cooperative dilemmas for people from different backgrounds. For instance, a study of fifteen nonindustrialized cultures found that the payoffs to cooperation in everyday life were positively correlated with the amount of economic game cooperation in each society (Henrich et al., 2005). An experimental demonstration of the same phenomenon comes from Study 2 of Stagnaro et al. (2017), in which participants began by playing several rounds of a public goods game. In some conditions, stingy contributions were punished, whereas in the control condition participants could behave selfishly with impunity. Participants who had been "enculturated" in the punishment version of the public goods game were later more cooperative in a second economic game that did not feature punishment.

If initial behavior in laboratory studies is shaped by a spillover from everyday life, then behavior at the end of experiments - that is, after a period of adjustment to the local incentivesshould be more reflective of participants' underlying goals (Binmore, 1999). For example, one 
cross-cultural study recruited samples from 16 different societies to play an iterated public goods game. Although there was considerable variation in average contribution level across societiesperhaps reflecting society-level variation in civic norms - all samples experienced a decline in cooperation across rounds, consistent with a pancultural desire to learn how to increase one's own income (Herrmann, Thöni, \& Gächter, 2008).

In a longitudinal demonstration of spillover (and subsequent adjustment), McAuliffe, Forster, Pedersen, and McCullough (2018a) had participants play cooperation games with anonymous strangers and privately donate to charity on two separate occasions. People contributed about $20 \%$ less to charity and strangers on the second occasion, except in the one game they played in which cooperation can yield a personal profit, even when played only once with a stranger. The researchers argued that participants acted on cooperative habits from everyday life during the first session, but by the time they arrived at the second session had learned that nobody would thank them for behaving fairly or scold them for behaving selfishly. Consistent with this hypothesis, decisions at the first but not the second session were positively associated with self- and peer-reports of cooperative traits, which do reflect how people behave in everyday social interactions (McAuliffe, Forster, Pedersen, \& McCullough, 2018b).

If learning explains cooperation in novel situations, then the ability to learn quickly should facilitate acting in accordance with one's true preferences during anonymous, one-shot interactions. Indeed, Burton-Chellew et al. (2016) found that free-riders were the only subset of participants who reliably understood that cooperation did not maximize personal income in the public goods game. Another study revealed that Japanese adults who never shared money across multiple one-shot economic games possessed more skill in understanding social situations (Yamagishi, Li, Takagishi, Matsumoto, \& Kiyonari, 2014). Finally, Barreda-Tarrazona, 
Jaramillo-Gutiérrez, Pavan, and Sabater-Grande (2017) reported that reasoning ability is associated with less cooperation in one-shot prisoner's dilemma games, and with more cooperation in repeated prisoner's dilemmas with the same partner. Both patterns are optimal: In the one-shot game there is no self-interested reason to cooperate, whereas in the repeated game inducing a long-term partner to cooperate in earlier rounds is essential to securing the benefits of cooperation in later rounds.

Learning, it should be noted, does not inevitably facilitate selfishness. Instead, an increased appreciation of a situation's incentives should increase alignment between people's behavior and their goals, whatever those goals happen to be. For example, most people who are forced to reflect on the one-shot, anonymous nature of the public goods game reduce their contributions (Rand, 2016). However, Grossmann, Brienza, and Bobocel (2017) found that participants who scored highly on a measure of wisdom still contributed in a public goods game even after pondering its incentive structure. The authors interpreted this finding as evidence that wise deliberation activated fairness principles that were more highly valued than earning extra money in a study. Another example of learning to achieve unselfish ends comes from Lockwood, Apps, Valton, Viding, and Roiser (2016), who had participants learn over several trials which of two choice options was associated with a monetary reward for either the self or another person. Overall, participants minimized prediction errors much more quickly when the rewards went to the self. However, the discrepancy in speed between learning on behalf of the self and another person was smaller for people higher in trait empathy, suggesting that empathic individuals are motivated to learn how to help others.

\section{Learning to Trust}


So far, we have discussed learning in the context of deciding whether cooperation is in one's interest. However, would-be cooperators still need to decide whom they can trust to reciprocate. Mistakenly cooperating with an untrustworthy partner is costly, but prediction errors are also opportunities to learn the subtle cues that predict cooperation. A recent experiment shows that humans are quick to pick up on correlates of trustworthiness (FeldmanHall, Dunsmoor, Tompary, Hunter, Todorov, \& Phelps, 2018). In this study, participants played several rounds of the Trust Game, an anonymous one-shot game in which an "investor" can transfer windfall money to a trustee. Transferred money is multiplied by a constant greater than one; then the trustee decides how much money to send back to the investor. A trustworthy trustee would send back more than they were sent, whereas an untrustworthy trustee would send back less money than they were sent. Participants acted as investor for three different trustees whose faces were revealed in pictures while participants made decisions about how much money to send. The trustees varied widely in how much money they returned, which gave participants an opportunity to associate different types of faces with different levels of trustworthiness. Participants generalized this association to a second set of trustees, whose faces were morphed to look like one of the faces from the first set. Specifically, participants preferred to send more to those who looked like generous trustees from the earlier games, and less to those who looked similar to the stingy ones.

Generalization of trust occurs beyond the laboratory, and indeed appears to account for individual differences in generalized trust (i.e., a belief that others are benevolent). In a study of Chinese CEOs, Nee, Holm, and Opper (2018) found that reliance on well-established business relationships was positively associated with choosing to play a trust game with a stranger rather than investing their money in a random lottery. Similarly, U.S. survey data revealed that 
socializing with friends, relatives and neighbors across time-points predicted increased generalized trust, and not vice versa (Glanville, Andersson, \& Paxton, 2013). Overall, people appear to use the quality of their own long-term relationships as a reference point for new relationships.

One might contend that generalized trust reflects an overgeneralization of one's own rewarding social relationships. However, high trusters are not gullible fools: On the contrary, a willingness to interact with unknown others allows high trusters to cultivate an ability to quickly pick up on cues that a new social partner is not trustworthy. In contrast, low trusters miss out on new, profitable relationships with trustworthy others and are slow to pull out of existing relationships in which they are exploited (Yamagishi, Kikuchi, \& Kosugi, 1999).

\section{Conclusion}

Experimental economists have long emphasized the role that learning plays in social decision-making (e.g., Binmore, 1999). However, cooperation researchers have only recently incorporated the role of learning into their studies. An important lesson from the research reviewed here is that people's behavior in any one situation is not necessarily a direct read-out of how selfish or altruistic they are, especially if the situation's incentives differ from what they normally encounter in everyday life. Overall, the learning perspective teaches us that explaining cooperation is not only a matter of understanding people's personalities and the situations they are in, but also what their past social interactions lead them to expect in novel social interactions. 


\section{References}

Barreda-Tarrazona, I., Jaramillo-Gutiérrez, A., Pavan, M., \& Sabater-Grande, G. (2017). Individual characteristics vs. experience: An experimental study on cooperation in prisoner's dilemma. Frontiers in Psychology, 8:596. doi: 10.3389/fpsyg.2017.00596

Binmore, K. (1999). Why experiment in economics? The Economic Journal, 109(453), 16-24.

Burton-Chellew, M. N., El Mouden, C., \& West, S. A. (2016). Conditional cooperation and confusion in public-goods experiments. Proceedings of the National Academy of Sciences, 113(5), 1291-1296.

Burton-Chellew, M. N., El Mouden, C., \& West, S. A. (2017). Social learning and the demise of costly cooperation in humans. Proceedings of the Royal Society of London B: Biological Sciences, 284(1853), 20170067.

Burton-Chellew, M. N., \& West, S. A. (2013). Prosocial preferences do not explain human cooperation in public-goods games. Proceedings of the National Academy of Sciences, 110(1), 216-221.

Chaudhuri, A. (2011). Sustaining cooperation in laboratory public goods experiments: a selective survey of the literature. Experimental Economics, 14(1), 47-83.

FeldmanHall, O., \& Dunsmoor, J. E. (2018). Viewing adaptive social choice through the lens of associative learning. Perspectives on Psychological Science, 1745691618792261.

FeldmanHall, O., Dunsmoor, J. E., Tompary, A., Hunter, L. E., Todorov, A., \& Phelps, E. A. (2018). Stimulus generalization as a mechanism for learning to trust. Proceedings of the National Academy of Sciences, 115(7), E1690-E1697.

Glanville, J. L., Andersson, M. A., \& Paxton, P. (2013). Do social connections create trust? An examination using new longitudinal data. Social Forces, 92(2), 545-562. 
Grossmann, I., Brienza, J. P., \& Bobocel, D. R. (2017). Wise deliberation sustains cooperation. Nature Human Behaviour, 1(3), 0061.

Henrich, J., Boyd, R., Bowles, S., Camerer, C., Fehr, E., Gintis, H., ... \& Henrich, N. S. (2005). "Economic man" in cross-cultural perspective: Behavioral experiments in 15 small-scale societies. Behavioral and Brain Sciences, 28(6), 795-815.

Herrmann, B., Thöni, C., \& Gächter, S. (2008). Antisocial punishment across societies. Science, 319(5868), 1362-1367.

Houser, D. \& Kurzban, R. (2002). Revisiting kindness and confusion in public goods experiments. American Economic Review, 92, 1062-1069.

Krasnow, M. M., \& Delton, A. W. (2016). Are humans too generous and too punitive? Using psychological principles to further debates about human social evolution. Frontiers in Psychology, 7, 799.

Lockwood, P. L., Apps, M. A., Valton, V., Viding, E., \& Roiser, J. P. (2016). Neurocomputational mechanisms of prosocial learning and links to empathy. Proceedings of the National Academy of Sciences, 113(35), 9763-9768.

McAuliffe, W.H.B., Forster, D.E., Pedersen, E.J., \& McCullough, M.E. (2018a). Experience with anonymous interactions reduces intuitive cooperation. Nature Human Behaviour, 2, 909-914.

McAuliffe, W.H.B., Forster, D.E., Pedersen, E.J., \& McCullough, M.E. (2018b). Does cooperation in the laboratory reflect the operation of a broad trait? European Journal of Personality. https://doi.org/10.1002/per.2180

Nee, V., Holm, H. J., \& Opper, S. (2018). Learning to trust: From relational exchange to generalized trust in China. Organization Science, 29(5), 969-986. 
Raihani, N. J., \& Bshary, R. (2015). Why humans might help strangers. Frontiers in behavioral neuroscience, 9,39 .

Rand, D. G. (2016). Cooperation, fast and slow: Meta-analytic evidence for a theory of social heuristics and self-interested deliberation. Psychological Science, 27(9), 1192-1206.

Rand, D. G., Peysakhovich, A., Kraft-Todd, G. T., Newman, G. E., Wurzbacher, O., Nowak, M. A., \& Greene, J. D. (2014). Social heuristics shape intuitive cooperation. Nature communications, 5, 3677.

Stagnaro, M. N., Arechar, A. A., \& Rand, D. G. (2017). From good institutions to generous citizens: Top-down incentives to cooperate promote subsequent prosociality but not norm enforcement. Cognition, 167, 212-254.

Yamagishi, T., Kikuchi, M., \& Kosugi, M. (1999). Trust, gullibility, and social intelligence. Asian Journal of Social Psychology, 2(1), 145-161.

Yamagishi, T., Li, Y., Takagishi, H., Matsumoto, Y., \& Kiyonari, T. (2014). In search of Homo economicus. Psychological Science, 25(9), 1699-1711. 


\section{Recommended Reading}

Raihani \& Bshary, 2015. (See references.) An overview of competing evolutionary explanations for why humans help others who they are unlikely to interact with again.

Binmore, K. (1999). (See references.) An early review of experimental economics research suggesting that participants' true economic preferences do not emerge until they have had experience with the laboratory task at hand.

Fehr, E., \& Schurtenberger, I. (2018). Normative foundations of human cooperation. Nature Human Behaviour, 2(7), 458. A recent articulation of the view that conditional cooperation reflects unselfish motives (as opposed to the view advanced here, which is that conditional cooperation is an artefact of learning how to maximize one's own income).

FeldmanHall, O., \& Dunsmoor, J. E. (2018). (See references.) A review of operant and Pavlovian learning processes that affect cooperation decisions.

Bear, A., \& Rand, D. G. (2016). Intuition, deliberation, and the evolution of cooperation. Proceedings of the National Academy of Sciences, 113(4), 936-941. A simple model that describes how either natural selection or social learning can favor humans who typically do not consider a situation's incentive structure before choosing to cooperate. 